\title{
AN ALL-DIGITAL, TIME-GATED 128X128 SPAD ARRAY FOR ON-CHIP, FILTER-LESS FLUORESCENCE DETECTION
}

\author{
Y. Maruyama and E. Charbon \\ Circuits and Systems, Delft University of Technology, Delft, The Netherlands
}

\begin{abstract}
This paper presents an on-chip, filter-less fluorescence detector using an all-digital, time-resolved imager based on a CMOS single-photon detector array with limited in situ processing. The device comprises an array of pixels capable of detecting single photons integrated with time gating circuitry and a 1-bit memory. The sensor allows on-chip, filter-less fluorescence detection and fluorescence lifetime imaging microscopy (FLIM) for micro total analysis systems ( $\mu$-TAS) and point-of-care testing (POCT) applications. The sensor was tested as an on-chip optical DNA detector using Cy5 labeled DNA molecules directly placed on the sensor surface.
\end{abstract}

\section{KEYWORDS}

CMOS, SPAD, TCSPC, Fluorescence detection

\section{INTRODUCTION}

The performance advantages of miniaturization in Micro-Total Analysis Systems ( $\mu$-TAS), such as rapid reaction and extreme reduction of sample volume, have been studied extensively with the specific goal of creating advanced tools for chemistry and the life sciences [1].

Intermolecular reactions, such as DNA hybridization, have been analyzed based on fluorescence detection employing an excitation light source and expensive optical components such as lenses and filters. Needless to say, a florescence microscope is standard equipment in life science, chemistry, and biochemistry. This is because 2D fluorescence images and image sequences produce a wealth of information all at once that are invaluable to researchers interested in the details of intraand inter-cellular reactions and dynamics. Information such as sample position, quantity, and 2D time-course analysis are the main stream of data used in $\mu$-TAS.

There are several approaches targeting on-chip fluorescence detection based on CMOS image sensors. The conventional approach is to use an on-chip optical filter to separate the excitation light from the faint fluorescence signal [2]. However, the limit of detection (LOD) of such on-chip fluorescence detection systems is determined by the nature of the filtering material, such as photoresist-based color filters. To overcome this issue, a new technique has been proposed and realized based on photon absorption depth difference in silicon [3][4]. However, a fluorescence 2D imaging device based on this method has not yet been reported.

In this paper we proposed an on-chip, filter-less fluorescence detector using an all-digital, time-resolved CMOS imager. In the proposed chip, time-resolved optical detection is achieved via an integrated single-photon avalanche diode (SPAD) based on [5][6]. Figure 1 shows the system block diagram. The array consists of $128 \times 128$ SPAD pixels which are read out in rolling shutter mode; the digital data is serially sent to an acquisition board for further processing. Time-correlated single-photon counting (TCSPC) is achieved by sliding-time-gating techniques [7], whereby the time window is controlled by a combination of an on-chip 600ps delay line and an off-chip, FPGA-based 200ps delay line. Thanks to this technique, it is possible to remove the excitation pulse from the integration window.

\section{CHIP ARCHTECURE}

Figure 2 shows the pixel architecture. Time gating is achieved by activating and deactivating the SPAD; turning on $T_{\text {recharge }}$ quickly brings the SPAD to operating conditions, while turning on $T_{\text {spadoff }}$ deactivates it by lowering the SPAD bias below breakdown. The voltage change caused by the avalanche is captured by an in-pixel NMOS 1-bit memory when the avalanche happened during $T_{\text {gate }}$ is on. The sliding-time-gating window is globally applied to all the pixels simultaneously and it is delayed with respect to the laser source by a fix amount of time; the process of laser illumination followed by gated exposure is repeated 16,384 times, after which the content of all the pixels is read out in rolling shutter mode. During readout the state of a SPAD is held by the 1-bit memory, thus no data contamination, signal droop, or readout errors are possible. The overall integration time of each pixel during a frame is $409 \mu \mathrm{s}$, while only the first successful detection is recorded. This is acceptable, since the sensor is intended for photon-starved operation, with fluorescence efficiencies of the order of $10^{-4}-10^{-6}$. Figure 3 illustrates the time diagram of a frame, comprising multiple illumination-and-exposure cycles and a single, final readout-and-reset cycle. The in-pixel 1-bit memory is reset $25 \mathrm{~ns}$ after readout, thus minimizing the dead time between frames. A micrograph of the sensor chip and a photograph of the entire system are shown in Figure 4. The chip, fabricated in a $0.35 \mu \mathrm{m}$ high-voltage standard CMOS process, has a total area of $20.5 \mathrm{~mm}^{2}$. The pixel pitch is $25 \mu \mathrm{m}$. The column decoder is used to serialize the data coming from the array onto two serial channels that are sent off-chip at $40 \mathrm{Mbps}$. A Spartan-3 FPGA board and a custom made PCB were used to build the ancillary electronics needed to operate the sensor system.

\section{RESULTS}


The sensor was first characterized in terms of its noise performance. In Figure 5 the dark count rate (DCR) cumulative probability is plotted for an excess bias voltage of $3 \mathrm{~V}$ at room temperature; with a median DCR of $78 \mathrm{~Hz}$, this sensor is one of the quietest CMOS SPAD arrays ever reported. Figure 6 shows the raw and median filtered fluorescence image of a pine pollen grain sample (Mixed pollen grains, Carolina Biological Supply Company, NC, USA) taken by using a fluorescence microscope (BX51IW, Olympus, Japan). Only $0.82 \%$ of the pixels have high noise, as shown in Figure 6(a), and can easily be removed using e.g. median filtering techniques, as shown in Figure 6(b).

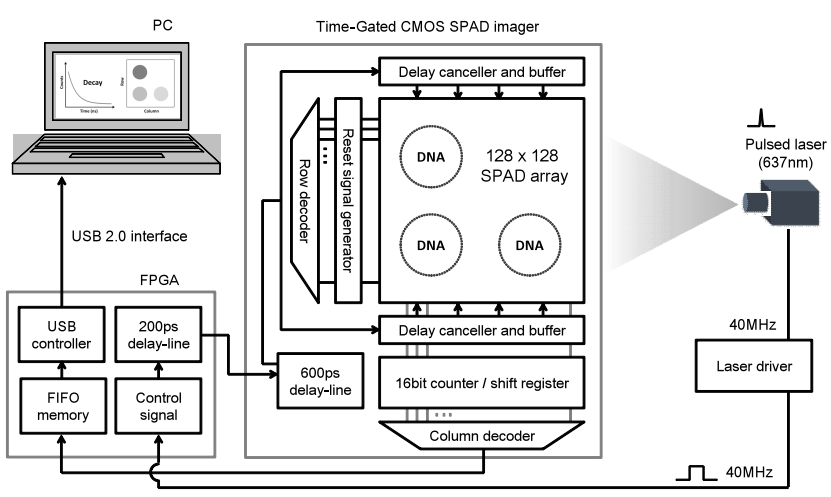

Figure 1: Block diagram of the proposed sensor. Time-resolved imaging is achieved via a sliding-time-gating scheme; the necessary signals are generated by a combination of an on-chip 600ps delay line and an off-chip 200ps delay line. The system is operated at $40 \mathrm{MHz}$, synchronized with the laser clock. The frame data are stored in FIFO memory on the FPGA board and read out through a USB 2.0 interface module.

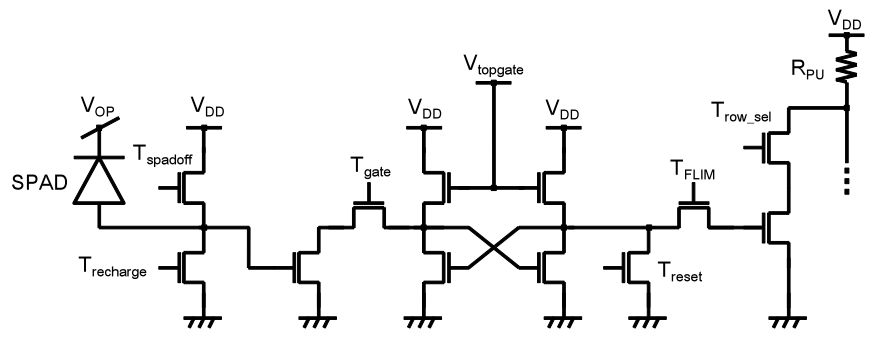

Figure 2: Schematic diagram of the pixel. Time gating is achieved through $T_{\text {spadoff, }} T_{\text {recharge }}$ and $T_{\text {gate }}$. The pixel also includes reset mechanisms of the 1-bit memory, as well as a pull down to transfer the memory content to the column. $V_{D D}$ and $V_{\text {topgate }}$ are $2.5 \mathrm{~V}$ and $2.0 \mathrm{~V}$, respectively.

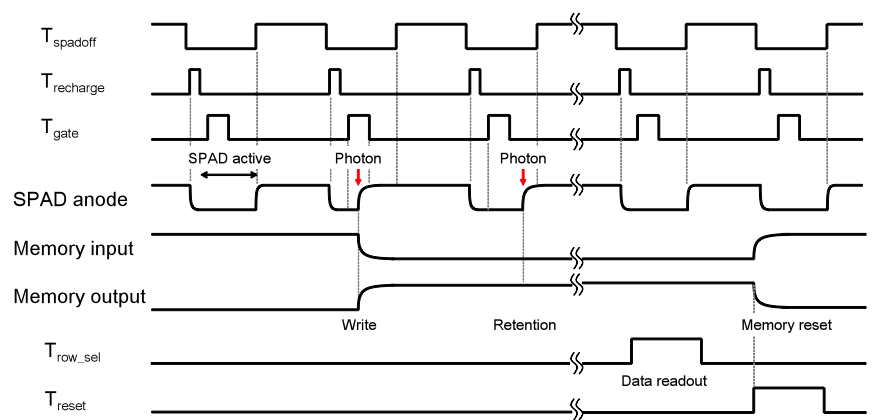

Figure 3: Timing diagram of the sliding-time-gating photon detection. Only the first avalanche event is stored in the in-pixel 1-bit memory during the frame integration time of $409 \mu \mathrm{s}$. The memory state is reset $25 \mathrm{~ns}$ after data readout.

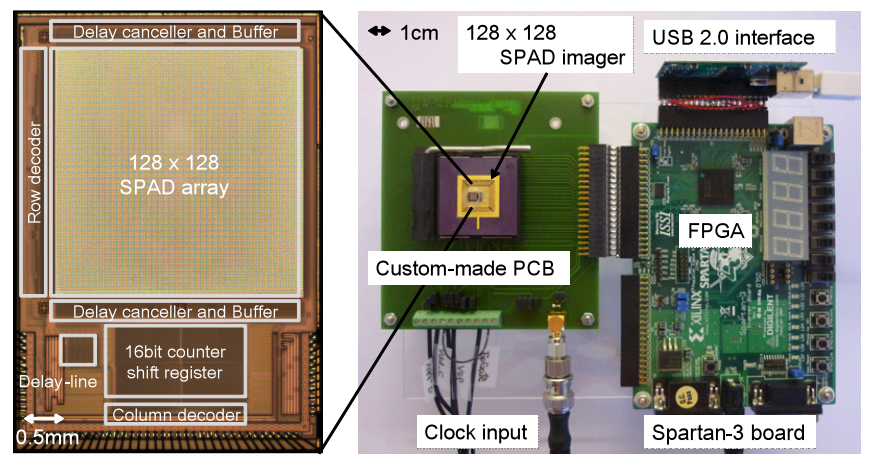

Figure 4: Micrograph of the sensor chip and photograph of the complete detection system.

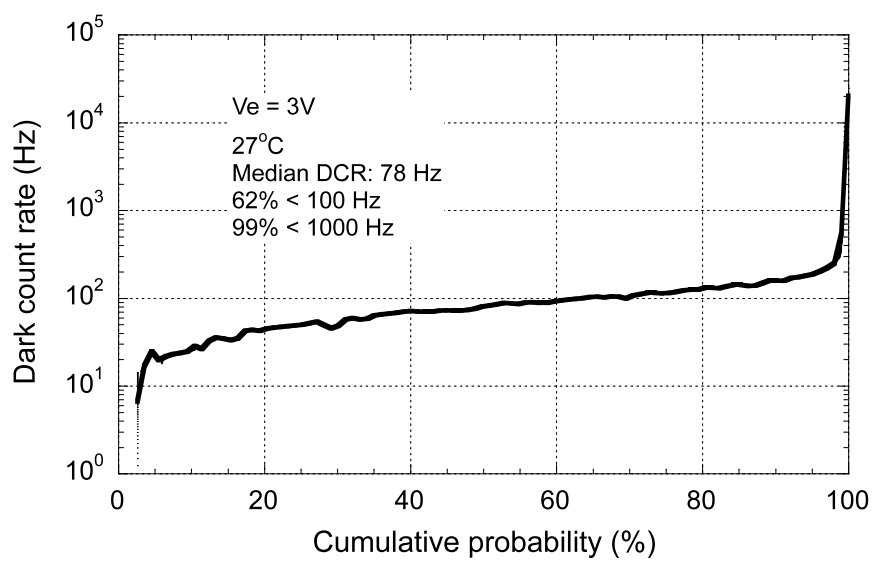

Figure 5: Dark count rate (DCR) cumulative probability for $3 V$ excess bias at room temperature. The number of noisy pixel is notably lower than that of other SPAD imagers fabricated in the same process [8].

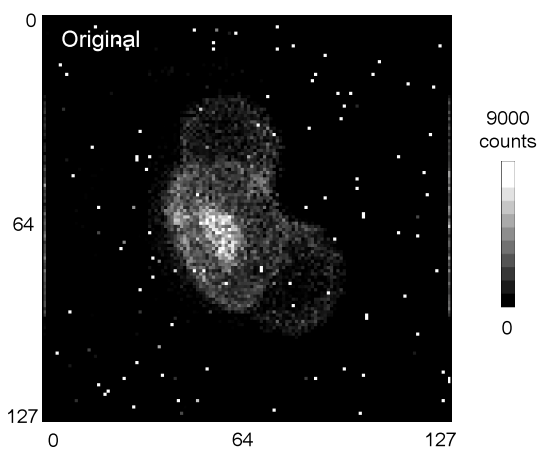

(a) $\ldots$

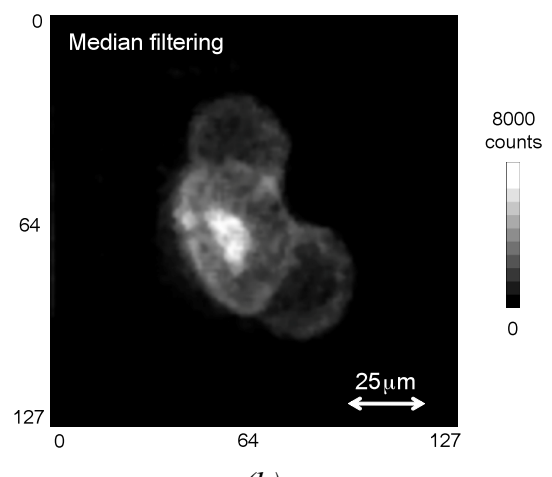

(b) ...

Figure 6: Fluorescence image of a pine pollen grain captured at a total integration time (multiple frames) of $585 \mathrm{~ms}$. (a) Raw image. 134 pixels out of 16384 have high noise as shown by 
white dot. (b) After median filtering (a noise reduction method widely used in signal processing) noisy pixels are removed.

The chip was subsequently tested as an on-chip, optical filter-less DNA sensor based on fluorescence detection. To avoid short circuits while handling aqueous samples, IO pads and bonding wires were covered with polydimethylsiloxane (PDMS), as shown in Figure 7. Three different concentrations, $36 \mu \mathrm{M}, 18 \mu \mathrm{M}$, and $9 \mu \mathrm{M}$, of Cy5 linked oligonucleotide (30mer) were placed on the sensor surface. The volume of each spot was 200nl, spread over approximately 2000 pixels.

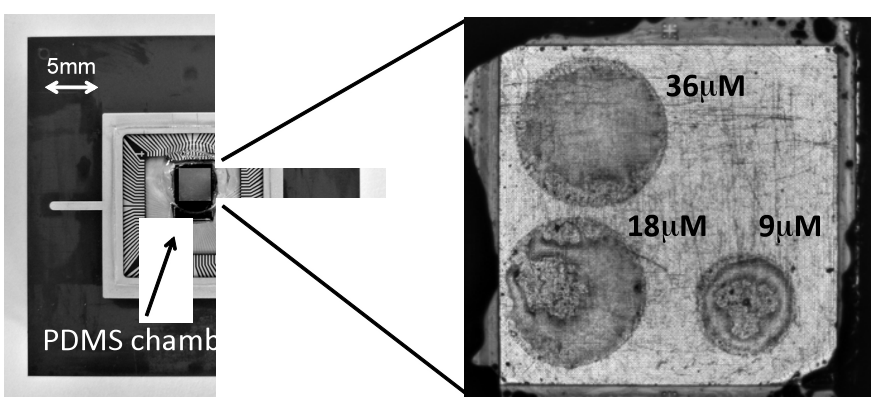

Figure 7: A PDMS (SYLGARD 184, Dow Corning Corp., USA) chamber and aqueous samples containing Cy5 labeled oligonucleotide. The DNA sample (Jena Bioscience, GmbH, Germany) was premixed and placed on the sensor surface.

Fluorescence lifetime histograms were obtained from 3ns after the laser pulse until 9.6ns, as shown in Figure 8 . The gate window width and time step were $3.6 \mathrm{~ns}$ and 200ps, respectively. The inset shows the response to optical excitation with a median FWHM jitter of 230ps over the entire array. Figure 9 shows the fluorescence 2D image from the DNA spots. The 2D image was obtained by accumulating all histogram bins over the range of interest. In Figure 10 the photon count rate increment of 502 counts $/ \mu \mathrm{M}$ was observed on average over 100 pixels in each spot. An LOD $(3 \sigma)$ of $14.6 \mu \mathrm{M}\left(8.8 \times 10^{8}\right.$ molecules per pixel) was estimated using the average standard deviation of 100 pixels in the reference area. The pixel-to-pixel variation is very high due to the non-uniform distribution of aqueous samples, as expected from Figure 7. Thus, the LOD can be improved by applying the proper sensor surface treatment, based for example on an ethylene glycol-silane monolayer [9]. The performance of the sensor is summarized in Table 1.

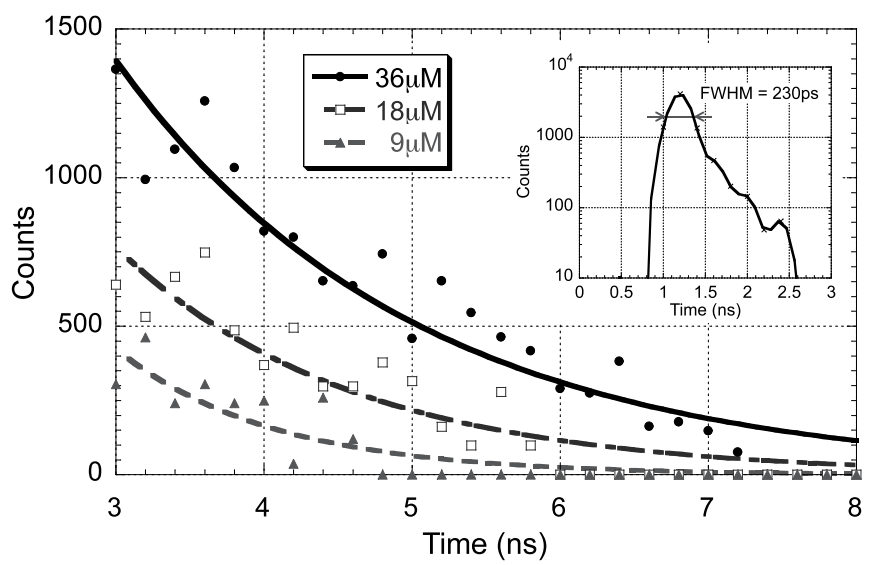

Figure 8: Histogram of photon counts from each DNA spot during time gating. The inset shows a median FWHM jitter of 230ps for the entire array. The excitation source is based on a pulsed laser diode (Advanced Laser Diode System, GmbH, Germany) with a wavelength of $637 \mathrm{~nm}$ and a pulse width of 40ps (FWHM). The total integration time of each histogram bin is $175 \mathrm{~ms}$ (multiple frames).

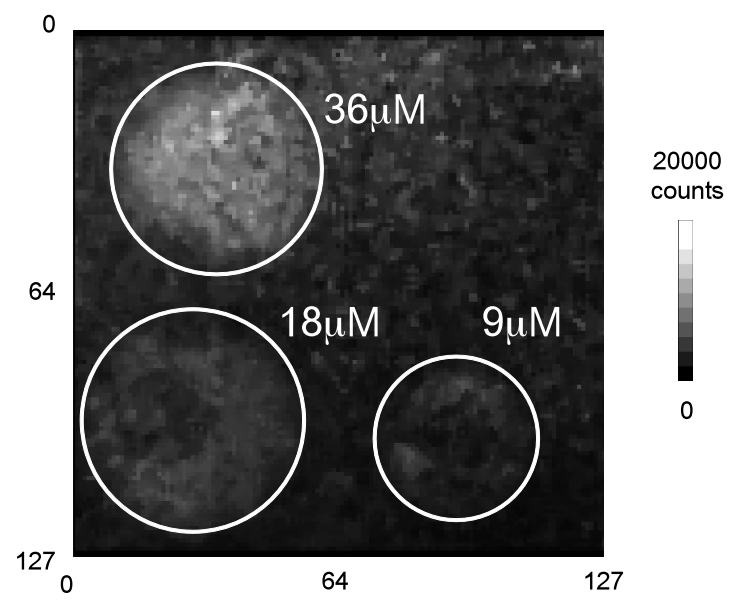

Figure 9: On-chip, filter-less fluorescence detection: the fluorescence label is Cy5 ( $\left.\lambda_{\text {ex }}=643 \mathrm{~nm}, \lambda_{\text {em }}=667 \mathrm{~nm}\right)$, a $637 \mathrm{~nm}$ pulsed laser is used for excitation light source.

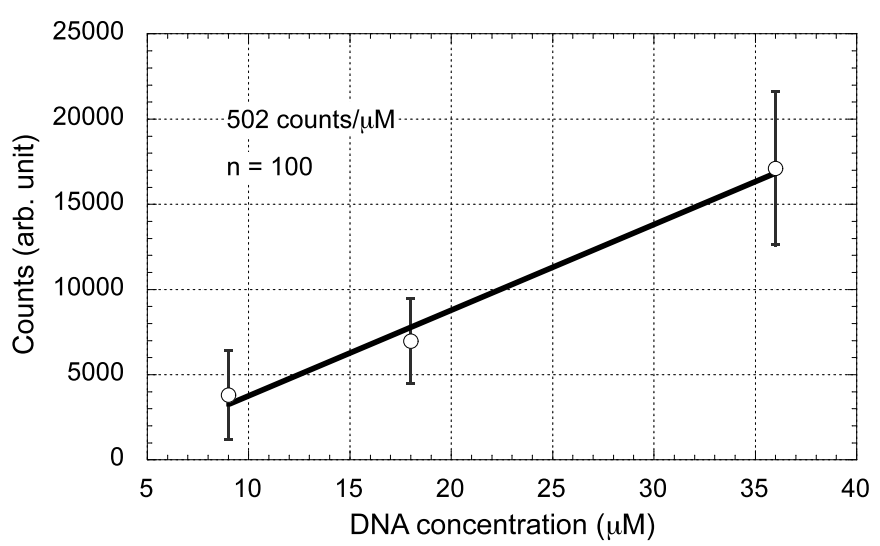

Figure 10: Integrated photon count rate. The LOD of $14.6 \mu M$ was measured without using optical filter.

Table 1. Performance summary for the image sensor.

\begin{tabular}{|c|c|c|}
\hline \multicolumn{1}{|c|}{ Parameter } & Value & Unit \\
\hline Array size & $128 \times 128$ & \\
\hline
\end{tabular}




\begin{tabular}{|l|c|c|}
\hline Pixel size & $25 \times 25$ & $\mu \mathrm{m}^{2}$ \\
\hline Fill factor & 4.5 & $\%$ \\
\hline Median DCR @ $\mathrm{V}_{\mathrm{e}}=3 \mathrm{~V}$ & 78 & $\mathrm{~Hz}$ \\
\hline Photon detection probability @ $\mathrm{V}_{\mathrm{e}}=3 \mathrm{~V}$ & $20 @ 465 \mathrm{~nm}$ & $\%$ \\
\hline Detection limit of DNA (LOD) & 14.6 & $\mu \mathrm{M}$ \\
\hline Time resolution (LSB) & 200 & $\mathrm{ps}$ \\
\hline Measurement range & 9.6 & $\mathrm{~ns}$ \\
\hline Jitter (FWHM) & 230 & $\mathrm{Ps}$ \\
\hline Operation frequency & 40 & $\mathrm{MHz}$ \\
\hline Frame rate & 2441 & $\mathrm{fps}$ \\
\hline Total IO bandwidth & 40 & $\mathrm{Mbps}$ \\
\hline Power consumption @ $\mathrm{V}_{\mathrm{dd}}=2.5 \mathrm{~V}$ & 363 & $\mathrm{~mW}$ \\
\hline Photo response non uniformity (PRNU) & 3.5 & $\%$ \\
\hline
\end{tabular}

\section{CONCLUSIONS}

An all-digital, time-gated CMOS SPAD imager was proposed and characterized as an on-chip, filter-less DNA detector. Using the sliding-time-gating technique, the photoresponse of labeled DNA was evaluated when excitated by a laser pulse. The excitation was removed by proper selection of the range of interest and faint fluorescence signals were successfully observed, thereby achieving an LOD of $14.6 \mu \mathrm{M}\left(8.8 \times 10^{8}\right.$ molecules per pixel) without using any optical filters. The technique realized here offers a new CMOS based experimental platform for $\mu$-TAS and POCT systems.

\section{ACKNOWLEDGEMENTS}

The authors would like to thank to P. Brühlmeier at École polytechnique fédérale de Lausanne (EPFL) for PCB layout and his valuable advices. The authors are also grateful to M. W. Fishburn at Delft University of Technology for meaningful discussions.

\section{REFERENCES}

[1] M. A. Burns, B N. Johnson, S. N. Brahmasandra, K. Handique, J. R. Webster, M. Krishnan, T. S. Sammarco, P. M. Man, D. Jones, D. Heldsinger, C. H. Mastrangelo, D. T. Burke "An Integrated Nanoliter DNA Analysis Device”, Science, 282, 484, 1998.

[2] A. Tagawa, M. Mitani, H. Minami, T. Noda, K. Sagawa T. Tokuda, and J. Ohta, "Complementary Metal Oxide Semiconductor Based Multimodal Sensor for In vivo Brain Function Imaging with Function for Simultaneous Cell Stimulation", Jpn. Appl. Phys. 49, 04DL02, 2010.

[3] Y. Maruyama, K. Sawada, M. Ishida, "Multiwavelength Photosensor for On-Chip Real-Time Monitoring of Fluorescence and Turbidity", Jpn. J. Appl. Phys., 48, 067003, 2009.

[4] H. Nakazawa, H. Ishii, M. Ishida, and K. Sawada, "A Fused $\mathrm{pH}$ and Fluorescence Sensor Using the Same Sensing Area", Applied Physics Express, Vol. 3, No. 4, 2010.

[5] C. Niclass, A. Rochas, P.A. Besse, and E. Charbon, "Design and Characterization of a CMOS 3-D Image Sensor based on Single Photon Avalanche Diodes", IEEE Journal of Solid-State Circuits, Vol. 40, N. 9, pp. 1847-1854, Sep. 2005.

[6] C. Niclass, M. Sergio, E. Charbon, "A single-photon avalanche diode array fabricated in $0.35 \mathrm{um}$ CMOS and based on an event-driven readout for TCSPC experiments", SPIE Optics East, Oct. 2006.

[7] D. Stoppa, D. Mosconi, L. Pancheri, and L. Gonzo, "Single-Photon Avalanche Diode CMOS Sensor for Time-Resolved Fluorescence Measurements", IEEE Sensors Journal, Vol. 9, No. 9, Sep. 2009.

[8] C. Niclass, Ph.D. dissertation no. EPFL, Switzerland, Reprint: Hartung-Gorre Verlag, ISBN-10: 3-86628-220-6, 2008.

[9] S. Carrara, A. Cavallini, Y. Maruyama, E. Charbon, G. D. Micheli, "A new ethylene glycol-silane monolayer for highly-specific DNA detection on Silicon Chips”, Surface Science, 604, 71-74, 2010.

\section{CONTACT}

Y. Maruyama, tel: +31-15-27-83663;

y.maruyama@tudelft.nl 